\title{
Proof of a Conjecture of Erdős on triangles in set-systems
}

\author{
Dhruv Mubayi * Jacques Verstraëte ${ }^{\dagger}$
}

November 11, 2005

\begin{abstract}
A triangle is a family of three sets $A, B, C$ such that $A \cap B, B \cap C, C \cap A$ are each nonempty, and $A \cap B \cap C=\emptyset$. Let $\mathcal{A}$ be a family of $r$-element subsets of an $n$-element set, containing no triangle. Our main result implies that for $r \geq 3$ and $n \geq 3 r / 2$, we have $|\mathcal{A}| \leq\left(\begin{array}{l}n-1 \\ r-1\end{array}\right)$. This settles a longstanding conjecture of Erdős [7], by improving on earlier results of Bermond, Chvátal, Frankl, and Füredi. We also show that equality holds if and only if $\mathcal{A}$ consists of all $r$-element subsets containing a fixed element.
\end{abstract}

Analogous results are obtained for nonuniform families.

\section{Introduction.}

Throughout this paper, $X$ is an $n$-element set. For any nonnegative integer $r$, we write $X^{(r)}$ for the family of all $r$-element subsets of $X$. Define $X^{(\leq r)}=\cup_{0 \leq i \leq r} X^{(i)}$ and $X^{(\geq r)}=\cup_{r \leq i \leq n} X^{(i)}$. For $\mathcal{A} \subset X^{(\leq n)}$ and $x \in X$, we let $\mathcal{A}_{x}=\{A \in \mathcal{A}: x \in A\}$.

A triangle is a family of three sets $A, B, C$ such that $A \cap B, B \cap C, C \cap A$ are each nonempty, and $A \cap B \cap C=\emptyset$. Let $f(r, n)$ denote the maximum size of a family $\mathcal{A} \subset X^{(r)}$ containing no triangle.

${ }^{*}$ Department of Mathematics, Statistics, \& Computer Science, University of Illinois at Chicago, 851 S. Morgan Street, Chicago, IL 60607-7045; email: mubayi@math.uic.edu

${ }^{\dagger}$ Department of Combinatorics and Optimization, University of Waterloo, Waterloo, ON N2L 3G1, Canada; E-mail: jverstra@math.uwaterloo.ca

2000 Mathematics Subject Classification: 05C35, 05C65, 05D05

Keywords: Extremal set theory, intersecting family, Baranyai's theorem

Accepted, Combinatorica 
A special case of Turán's theorem (proved by Mantel) implies that $f(2, n)=\left\lfloor n^{2} / 4\right\rfloor$. Motivated by this result, Erdős [7] asked for the determination of $f(r, n)$ for $r>2$, and conjectured that

$$
f(r, n)=\left(\begin{array}{l}
n-1 \\
r-1
\end{array}\right) \quad \text { for } \quad n \geq 3 r / 2 .
$$

(Actually, in [7] it is stated more as a question, and $n \geq 3 r / 2$ is not explicitly mentioned, but later, e.g. in $[3,10],(1)$ is referred to as a conjecture of Erdős'.)

This conjecture attracted quite a few researchers. It was proved by Chvátal [3] for $r=3$. In fact, he proved the more general statement that if $n \geq r+2 \geq 5, \mathcal{A} \subset X^{(r)}$, and $|\mathcal{A}|>\left(\begin{array}{c}n-1 \\ r-1\end{array}\right)$, then $\mathcal{A}$ contains $r$ sets $A_{1}, \ldots, A_{r}$ such that every $r-1$ of them have nonempty intersection, but $\cap_{i} A_{i}=\emptyset$. This configuration is also called an $(r-1)$-dimensional simplex. Chvátal generalized (1) as follows.

Conjecture 1 (Chvátal) Let $r \geq d+1 \geq 3, n \geq r(d+1) / d$ and $\mathcal{A} \subset X^{(r)}$. If $\mathcal{A}$ contains no $d$-dimensional simplex, then $|\mathcal{A}| \leq\left(\begin{array}{c}n-1 \\ r-1\end{array}\right)$. Equality holds only when $\mathcal{A}=X_{x}^{(r)}$, for some $x \in X$.

Recently Csákány and Kahn [6] gave a different proof of the $r=3$ case of (1) using Homology theory. Frankl [9] settled (1) for $3 r / 2 \leq n \leq 2 r$, and then Bermond and Frankl [2] proved (1) for infinitely many $n, r$, where $n<r^{2}$. About five years later, Frankl [10] settled (1) for $n>n_{0}(r)$, where $n_{0}(r)$ is an unspecified but exponentially growing function of $r$. In 1987, Frankl and Füredi [11] proved Conjecture 1 for $n>n_{0}(r)$. Frankl [10] had earlier verified Conjecture 1 for $(d+1) r / d \leq n<2 r$, using Katona's permutation method. Thus both (1) and Conjecture 1 remained open in the range $2 r \leq n<n_{0}(r)$, where $n_{0}(r)$ is exponential in $r$. Also, the uniqueness of the extremal configuration remained open for $3 r / 2 \leq n<n_{0}(r)$ in both (1) and Conjecture 1 . Our main result settles (1) for all $n \geq 3 r / 2$ while also characterizing the extremal examples. A nontrivial intersecting family of size $d+1$ is a family of $d+1$ distinct sets $A_{1}, \ldots, A_{d+1}$ that have pairwise nonempty intersection, but $\cap_{i} A_{i}=\emptyset$.

Theorem 2 Let $r \geq d+1 \geq 3$ and $n \geq(d+1) r / d$. Suppose that $\mathcal{A} \subset X^{(r)}$ contains no nontrivial intersecting family of size $d+1$. Then $|\mathcal{A}| \leq\left(\begin{array}{c}n-1 \\ r-1\end{array}\right)$. Equality holds if and only if $\mathcal{A}=X_{x}^{(r)}$ for some $x \in X$.

Note that the special case $d=2$ above implies (1). Every $d$-dimensional simplex is a nontrivial intersecting family of size $d+1$, and in this sense Theorem 2 can be thought of as a solution to a weakening of Conjecture 1 . 
A hypergraph $\mathcal{F}$ satisfies $H_{d}$, the Helly property of order $d$, if every subfamily of $\mathcal{F}$ with empty intersection contains a subcollection of at most $d$ sets with empty intersection. A related problem is to determine the maximum size of an $\mathcal{F} \in X^{(r)}$ that satisfies $H_{d}$. Theorem 2 implies that for $d=2$, such an $\mathcal{F}$ satisfies $|\mathcal{F}| \leq\left(\begin{array}{c}n-1 \\ r-1\end{array}\right)$, however, stronger results for this problem were obtained by several authors (see Bollobás and Duchet $[4,5]$, Tuza [15, 16], and Mulder [13]).

The proof of Theorem 2 actually gives a little more: we may allow $r \leq d \leq \min \left\{\left(\begin{array}{c}2 r-2 \\ r-1\end{array}\right),\left(\begin{array}{c}n-1 \\ r-1\end{array}\right)\right\}$. Theorem 2 is not valid when $r=3$ and $d \geq 10$ however, as the next result attests (see Section 4):

Theorem 3 Let $\mathcal{A} \subset X^{(3)}$ contain no non-trivial intersecting family of size $d+1 \geq 8$. Then

$$
|\mathcal{A}| \leq\left(\left\lfloor\frac{(d+2)}{3}\right\rfloor^{-1}+\left\lfloor\frac{(d+3)}{3}\right\rfloor^{-1}+\left\lfloor\frac{(d+4)}{3}\right\rfloor^{-1}\right)^{-1}\left(\begin{array}{l}
n \\
2
\end{array}\right) \leq \frac{1}{3}\left(\left\lceil\frac{d}{3}\right\rceil+\frac{1}{d+3}\right)\left(\begin{array}{l}
n \\
2
\end{array}\right) .
$$

Furthermore, for $d+1 \geq 11$ and infinitely many $n$, there exists such a family $\mathcal{A}$ with $|\mathcal{A}| \geq$ $\left(\frac{1}{3}\left\lceil\frac{d}{3}\right\rceil-\frac{1}{3}\right)\left(\begin{array}{l}n \\ 2\end{array}\right)$.

We conjecture that for $r \geq 4$ and $n$ sufficiently large, the phenomenon exhibited by Theorem 3 does not arise:

Conjecture 4 Let $r \geq 4, d \geq 2$, and let $\mathcal{A} \subset X^{(r)}$ contain no non-trivial intersecting family of size $d+1$. Then, provided $n$ is sufficiently large, $|\mathcal{A}| \leq\left(\begin{array}{c}n-1 \\ r-1\end{array}\right)$ with equality if and only if $\mathcal{A}=X_{x}^{(r)}$ for some $x \in X$.

The following table summarizes the above results for $r=3$ :

\begin{tabular}{|l|l|l|}
\hline Size & Lower Bound & Upper Bound \\
\hline \hline $3 \leq d+1 \leq 7$ & $\left(\begin{array}{c}n-1 \\
2\end{array}\right)$ & $\left(\begin{array}{c}n-1 \\
2\end{array}\right)$ \\
\hline$d+1=8$ & $\left(\begin{array}{c}n-1 \\
2\end{array}\right)$ & $\left(\begin{array}{c}n \\
2\end{array}\right)$ \\
\hline$d+1=9$ & $\left(\begin{array}{c}n-1 \\
2\end{array}\right)$ & $\frac{12}{11}\left(\begin{array}{c}n \\
2\end{array}\right)$ \\
\hline$d+1=10$ & $\left(\begin{array}{c}n-1 \\
2\end{array}\right)$ & $\frac{6}{5}\left(\begin{array}{c}n \\
2\end{array}\right)$ \\
\hline$d+1 \geq 11$ & $\frac{1}{3}\left(\left\lceil\frac{d}{3}\right\rceil-1\right)\left(\begin{array}{l}n \\
2\end{array}\right)$ & $\frac{1}{3}\left(\left\lceil\frac{d}{3}\right\rceil+\frac{1}{d+3}\right)\left(\begin{array}{c}n-1 \\
2\end{array}\right)$ \\
\hline
\end{tabular}

It would be interesting to determine the exact bounds for $d+1 \geq 11$. In the course of the proof of the lower bound in Theorem 3 , it is proved that a Steiner $(n, 3, k-1)$-system, when it exists, contains no non-trivial intersecting family of size $3 k+1$ whenever $k \geq 2$. We conjecture that this is the extremal family for $r=3$ and $k \geq 2$ : 
Conjecture 5 Let $n$ be sufficiently large and let $k \geq 2$. Let $\mathcal{A} \subset X^{(3)}$ contain no non-trivial intersecting family of size $3 k+1$, and suppose there exists a Steiner $(n, 3, k-1)$-system. Then $|\mathcal{A}| \leq \frac{1}{3}(k-1)\left(\begin{array}{l}n \\ 2\end{array}\right)$, with equality if and only if $\mathcal{A}$ is such a Steiner system.

Non-uniform families: It is natural to consider these extremal problems for families that are not uniform. Perhaps the most basic statement in this context is the analogue of the Erdös-Ko-Rado Theorem.

$$
\text { If } \mathcal{A} \subset X^{(\leq n)} \text { is intersecting, then }|\mathcal{A}| \leq 2^{n-1} \text {. }
$$

The non-uniform analogue of Erdös' conjecture about triangles in uniform families was asked by Erdős and proved by Milner [7].

Theorem 6 (Milner) Suppose that $\mathcal{A} \subset X^{(\leq n)}$ is triangle free. Then $|\mathcal{A}| \leq 2^{n-1}+n$.

Since Milner's proof has not been published, we give our own short proof of this result (see also Lossers [12]). Our proof also yields that equality holds if and only if $\mathcal{A}=X_{x}^{(\geq 2)} \cup X^{(1)} \cup\{\emptyset\}$ for some $x \in X$; this fact seems not to have been mentioned in the previous literature. We also prove the non-uniform analogue of Theorem 2 (see Section 4).

Theorem 7 Let $d \geq 2$ and $n>\log _{2} d+\log _{2} \log _{2} d+2$. Suppose that $\mathcal{A} \subset X^{(\leq n)}$ contains no non-trivial intersecting family of size $d+1$. Then $|\mathcal{A}| \leq 2^{n-1}+n$. Equality holds if and only if $\mathcal{A}=X_{x}^{(\geq 2)} \cup X^{(1)} \cup\{\emptyset\}$ for some $x \in X$.

If $n \leq\left\lfloor\log _{2} d\right\rfloor$, then trivially the bound $|\mathcal{A}| \leq 2^{n-1}+n$ in Theorem 7 does not hold. It can be shown that this remains true for $\left\lfloor\log _{2} d\right\rfloor+1$ and $\left\lfloor\log _{2} d\right\rfloor+2$. However, once $n>\left\lfloor\log _{2} d\right\rfloor+$ $\log _{2} \log _{2} d+2$, Theorem 7 applies. It would be interesting to determine if the $\log _{2} \log _{2} d$ term in Theorem 7 can be replaced by an absolute constant.

\section{Proof of Theorem 2.}

We use the notation $[n]=\{1, \ldots, n\}$ and $[a, b]=\{a, a+1, \ldots, b-1, b\}$. Let $\mathcal{A}$ be a family of $r$-sets with $|\mathcal{A}| \geq\left(\begin{array}{c}n-1 \\ r-1\end{array}\right)$, containing no non-trivial intersecting family of size $d+1$. We prove that $\mathcal{A}$ consists of all sets containing a fixed element of $X$. The proof, for $n \geq(d+1) r / d$, is split into three parts; 
Part I $\quad n<2 r$ and $n=k(n-r)+\ell$ with $k \in[2, d]$ and some $\ell \in[n-r-1]$,

Part II $\quad n<2 r$ and $n=k(n-r)$ with $k \in[3, d+1]$,

Part III $n \geq 2 r \geq 8$.

Note that for $(d+1) r / d \leq n \leq 2 r-1$, there exist $k \in[2, d]$ and $\ell \in[n-r-1]$ such that $n=k(n-r)+l$ or $n=(k+1)(n-r)$. Thus Parts I and II include all these values of $n$.

Part I uses Katona's permutation method, Part II uses Baranyai's Theorem [1] on partitioning $X^{(r)}$ into matchings, and in Part III we proceed by induction on $n$. Frankl [9] established the upper bound $|\mathcal{A}| \leq\left(\begin{array}{c}n-1 \\ r-1\end{array}\right)$ for $(d+1) r / d \leq n \leq 2 r-1$; however, it is substantially more difficult to establish the case of equality in Theorem 2, which we achieve in Parts I and II of our proof.

Part I: $n=k(n-r)+\ell$.

In this part, we consider the case $n<2 r$ and $n=k(n-r)+\ell$, for some $k \in[2, d]$ and $\ell \in[n-r-1]$. For convenience, let $X=[n]$ and fix a (cyclic) permutation $\pi$ of $X$. Let $Q_{i}$ denote the interval $\{i, i+1, \ldots, i+r-1\}$ (modulo $n$ ), and let $\mathcal{A}_{\pi}$ denote the subfamily of $\mathcal{A}$ consisting of those sets $A \in \mathcal{A}$ such that $\pi\left(Q_{i}\right)=A$ for some $i$ :

$$
\mathcal{A}_{\pi}=\left\{\pi\left(Q_{i}\right): \pi\left(Q_{i}\right) \in \mathcal{A}\right\}
$$

Claim 1. Let $\pi$ be any permutation. Then $\left|\mathcal{A}_{\pi}\right| \leq r$ with equality if and only if there exists $m$ such that

$$
\mathcal{A}_{\pi}=\left\{\pi\left(Q_{m}\right), \pi\left(Q_{m+1}\right), \ldots, \pi\left(Q_{m+r-1}\right)\right\}
$$

Proof. It is sufficient to prove Claim 1 for the identity permutation, since we may relabel $X$. Therefore $\mathcal{A}_{\pi}=\left\{Q_{i}: Q_{i} \in \mathcal{A}\right\}$. Without loss of generality, $Q_{n} \in \mathcal{A}_{\pi}$. For $j \in[n-r]$, let $P_{j}=\{i: i \equiv j(\bmod n-r)\} \cap[n]$, together with $\{n\}$ if $j \in[\ell+1, n-r]$. Thus $\left|P_{j}\right| \leq k+1 \leq d+1$. For each $j \in[n-r]$, there is an $i \in P_{j}$ such that $Q_{i} \notin \mathcal{A}_{\pi}$, otherwise $\bigcap_{i \in P_{j}} Q_{i}=\emptyset$. Thus $Q_{i} \notin \mathcal{A}_{\pi}$ for at least $n-r$ values of $i$, so $\left|\mathcal{A}_{\pi}\right| \leq r$.

Equality holds only if there is a unique $x_{j}$ such that $Q_{x_{j}(n-r)+j} \notin \mathcal{A}_{\pi}$ for all $j \in[n-r]$. We now show $x_{1} \geq x_{2} \geq \ldots \geq x_{n-r} \geq x_{1}-1$. Let us illustrate the proof of this fact using Figures 1 and 2 below, where $y_{j}=x_{j}(n-r)+j$, and the box $(i, j)$ represents the integer $(i-1)(n-r)+j$ : 
If $x_{j}<x_{j+1}$ for some $j \in[\ell]$, then, since $\ell \leq n-r-1$, the intersection of the $k+1$ intervals $Q_{(i-1)(n-r)+j}$, where $(i, j)$ is a shaded box in Figure 1 , is empty (this is the only place in Part I where we use $\ell \leq n-r-1$; the case $\ell=n-r-1$ is the content of Part II). This contradiction implies that $x_{j} \geq x_{j+1}$. In a similar way, $x_{j} \geq x_{j+1}$ for $j \in[\ell+1, n-r]$, using $Q_{n} \in \mathcal{A}_{\pi}$. Finally, if $x_{n-r}<x_{1}-1$, then the intersection of the intervals $Q_{(i-1)(n-r)+j}$, with $(i, j)$ a shaded box in Figure 2, is empty, a contradiction. This proves that $\mathcal{A}_{\pi}$ has the required form.

Without loss of generality, we assume that for the identity permutation $\iota, \mathcal{A}_{\iota}=\left\{Q_{1}, Q_{2}, \ldots, Q_{r}\right\}$.

Claim 2. For each permutation $\pi, \mathcal{A}_{\pi}=\left\{\pi\left(Q_{1}\right), \pi\left(Q_{2}\right), \ldots, \pi\left(Q_{r}\right)\right\}$.

Proof. Each permutation $\pi$ of $X \backslash\{r\}$ is a product of transpositions. Therefore it suffices to show that if $\tau$ is a transposition in which $r$ is a fixed point, then

$$
\mathcal{A}_{\tau}=\left\{\tau\left(Q_{1}\right), \tau\left(Q_{2}\right), \ldots, \tau\left(Q_{r}\right)\right\}
$$

Suppose that $\tau$ transposes $t$ and $t+1$, with $r \notin\{t, t+1\}$. Then Claim 1 implies that $\mathcal{A}_{\tau}=$ $\left\{\tau\left(Q_{m}\right), \tau\left(Q_{m+1}\right), \ldots, \tau\left(Q_{m+r-1}\right)\right\}$ for some $m \in[n]$. We show below that $m=1$.

Case 1. $n \notin\{t, t+1\}$ : Here $\tau\left(Q_{1}\right)=[r]=Q_{1} \in \mathcal{A}$, and $\tau\left(Q_{n}\right)=\{1, \ldots, r-1, n\}=Q_{n} \notin \mathcal{A}$. Therefore $m=1$. 
Case 2. $t+1=n$ : In this case $\tau\left(Q_{i}\right)=Q_{i} \in \mathcal{A}$ for each $i \in[r] \backslash\{n-r\}$. Consequently $\tau\left(Q_{n-r}\right) \in \mathcal{A}$ as well, and therefore $m=1$.

Case 3. $t=n$ : If $n<2 r-1$, then $\tau\left(Q_{r}\right)=Q_{r} \in \mathcal{A}$ and $\tau\left(Q_{r+1}\right)=Q_{r+1} \notin \mathcal{A}$. Therefore $m=1$. If $n=2 r-1$, then $\tau\left(Q_{i}\right)=Q_{i} \in \mathcal{A}$ for $i=2, \ldots, r-1$. This leaves the posibilities $m=1,2, n$. However, $\tau\left(Q_{r+1}\right)=Q_{r+1} \notin \mathcal{A}$, and $\tau\left(Q_{n}\right)=Q_{n} \notin \mathcal{A}$. Consequently, $\left\{\tau\left(Q_{1}\right), \tau\left(Q_{r}\right)\right\} \subset \mathcal{A}$ and $m=1$ again.

We now complete Part I. For each $A \in \mathcal{A}$, there are $\frac{1}{2} r !(n-r)$ ! families $\mathcal{A}_{\pi}$ containing $A$. The total number of cyclic permutations of $X$ is $(n-1) ! / 2$. By Claim $1,\left|\mathcal{A}_{\pi}\right| \leq r$ and therefore

$$
\frac{1}{2} r !(n-r) !|\mathcal{A}| \leq \frac{1}{2} r(n-1) !
$$

This establishes the upper bound $|\mathcal{A}| \leq\left(\begin{array}{c}n-1 \\ r-1\end{array}\right)$. By Claim 1, equality holds if and only if for every cyclic permutation $\pi$ of $X$, we have $\mathcal{A}_{\pi}=\left\{\pi\left(Q_{1}\right), \pi\left(Q_{2}\right), \ldots, \pi\left(Q_{r}\right\}\right.$. Set $x=r$. For any $A \subset(X \backslash\{x\})^{(r-1)}$, we may thus choose such a cyclic permutation $\pi$ so that $\pi\left(Q_{1}\right)=A \cup\{x\}$. Therefore $A \cup\{x\} \in \mathcal{A}$, and $\mathcal{A}=X_{x}^{(r)}$ is the required family.

$$
\text { Part II: } n=k(n-r) \text {. }
$$

The argument here is different to that of Part I; we use a result of Baranyai [1], stating that the family $X^{(r)}$ may be partitioned into perfect matchings when $r$ divides $n$. This result is only needed for the characterization of the extremal family $\mathcal{A}$. Recall that $\overline{\mathcal{A}}=\{X \backslash A: A \in \mathcal{A}\}$.

Claim 3. If $A \in X^{(n-r)} \backslash \overline{\mathcal{A}}$, then $(X \backslash A)^{(n-r)} \subset \overline{\mathcal{A}}$.

Proof. Pick $A^{\prime} \in(X \backslash A)^{(n-r)}$. We will show that $A^{\prime} \in \overline{\mathcal{A}}$. By Baranyai's Theorem, there is a partition of $X^{(n-r)}$ into perfect matchings $\mathcal{M}_{1}, \ldots, \mathcal{M}_{t}$ of size $k$, where $t=\frac{1}{k}\left(\begin{array}{c}n \\ n-r\end{array}\right)$. By relabelling $X$ if necessary, we may assume that $\mathcal{M}_{1} \supset\left\{A, A^{\prime}\right\}$. Since $\overline{\mathcal{A}}$ has no perfect matching, and $n=k r /(k-1)$,

$$
|\overline{\mathcal{A}}| \leq(k-1) t=\frac{k-1}{k}\left(\begin{array}{c}
n \\
n-r
\end{array}\right)=\frac{k-1}{k}\left(\begin{array}{c}
n \\
r
\end{array}\right)=\frac{k-1}{k} \frac{n}{r}\left(\begin{array}{c}
n-1 \\
r-1
\end{array}\right)=\left(\begin{array}{c}
n-1 \\
r-1
\end{array}\right) .
$$

Therefore $|\mathcal{A}|=|\overline{\mathcal{A}}|=\left(\begin{array}{c}n-1 \\ r-1\end{array}\right)$, and $\left|\overline{\mathcal{A}} \cap \mathcal{M}_{i}\right|=k-1$ for all $i$. Since $\mathcal{M}_{1} \supset\left\{A, A^{\prime}\right\}$ and $A \notin \overline{\mathcal{A}}$, we must have $A^{\prime} \in \overline{\mathcal{A}}$. Therefore Claim 3 is verified. 
We now complete the proof of Theorem 2 for $n=k(n-r)$. Let $\mathcal{B}=X^{(n-r)} \backslash \overline{\mathcal{A}}$. Then $n(\mathcal{B})=$ $\frac{k}{k-1} r \geq 2(n-r)$ as $k \geq 2$ and $n<2 r$. Furthermore, $\mathcal{B}$ is an intersecting family, by Claim 3 , and $|\mathcal{B}|=\left(\begin{array}{c}n \\ n-r\end{array}\right)-|\overline{\mathcal{A}}|=\left(\begin{array}{c}n-1 \\ n-r-1\end{array}\right)$. By Theorem $1, \mathcal{B}=X_{x}^{(n-r)}$ for some $x \in X$. This shows that $\mathcal{A}=X_{x}^{(r)}$, and Part II is complete.

\section{Part III: $n \geq 2 r$.}

Throughout Part III, we assume $r \geq 4$. Addition of technical details in Claim 3 in the proof below accommodates the case $r=3$. However, a short proof in this case was presented by weight counting techniques in Frankl and Füredi [11], which we revisit in Section 5.

We need the following notations.

For $\mathcal{A} \subset X^{(r)}$, let $V(\mathcal{A})=\bigcup_{A \in \mathcal{A}} A$ and $n(\mathcal{A})=|V(\mathcal{A})|$. For $Y \subset X$, we define $\mathcal{A}-Y=\{A \in \mathcal{A}$ : $A \cap Y=\emptyset\}$. We also write $\overline{\mathcal{A}}=\{X \backslash A: A \in \mathcal{A}\}$. The following five definitions and the associated notations will be used repeatedly throughout the paper:

Sum of Families. The sum of families $\mathcal{A}_{1}, \mathcal{A}_{2}, \ldots, \mathcal{A}_{t}$, denoted $\sum_{i} \mathcal{A}_{i}$, is the family of all sets in each $\mathcal{A}_{i}$. Note that $\sum \mathcal{A}_{i}$ may have repeated sets, even if none of the $\mathcal{A}_{i}$ have repeated sets.

Trace of a Set. The trace of a set $Y$ in $\mathcal{A}$ is defined by $\operatorname{tr}(Y)=\operatorname{tr}_{\mathcal{A}}(Y)=\{A \subset X: A \cup Y \in \mathcal{A}\}$. We define $\operatorname{tr}(\mathcal{A})=\sum_{x \in X} \operatorname{tr}(x)$.

Degree of a Set. The edge neighborhood of a set $Y$ is $\Gamma(Y)=\Gamma_{\mathcal{A}}(Y)=\{A \in \mathcal{A}: A \cap Y \neq$ $\emptyset$ and $A \neq Y\}$, and the degree of $Y$ is $\operatorname{deg}_{\mathcal{A}}(Y)=\left|\Gamma_{\mathcal{A}}(Y)\right|$. If $Y=\{y\}$, then we write $y$ instead of $\{y\}$, and $\operatorname{deg}_{\mathcal{A}}(y)=\left|\Gamma_{\mathcal{A}}(y)\right|=\left|\mathcal{A}_{y}\right|$.

The families $\mathcal{S}_{x}$ and $\mathcal{L}_{x}$. Let $\mathcal{A}$ be an $r$-uniform family of sets in $X$ and $x \in X$. Then we define

$$
\mathcal{S}_{x}=\{Y \in \operatorname{tr}(x):|\operatorname{tr}(Y)|=1\} \quad \text { and } \quad \mathcal{L}_{x}=\operatorname{tr}(x) \backslash \mathcal{S}_{x} .
$$

We write $\mathcal{S}=\sum_{x \in X} \mathcal{S}_{x}$ and $\mathcal{L}=\sum_{x \in X} \mathcal{L}_{x}=\operatorname{tr}(\mathcal{A}) \backslash \mathcal{S}$. Note that if $A \in \mathcal{L}_{x}$, then there exists $y \neq x$ such that $A \in \mathcal{L}_{y}$.

Paths and Connectivity. A path in $\mathcal{A}$ is a family $\mathcal{P}$ of sets $A_{1}, A_{2}, \ldots$ such that $A_{i} \cap A_{j} \neq \emptyset$ if and only if $|i-j| \leq 1$. Family $\mathcal{A}$ is connected if every pair of vertices in $V(\mathcal{A})$ is contained in some path in $\mathcal{A}$. A component of $\mathcal{A}$ is a maximal non-empty connected subfamily of $\mathcal{A}$.

We begin with the following simple lemma. Recall that $\mathcal{B}-S=\{A \in \mathcal{B}: A \cap S=\emptyset\}$. 
Lemma 8 Let $\mathcal{B}_{0}$ be a finite family of sets. Then there exist disjoint sets $S_{0}, S_{1}, \ldots, S_{t-1} \in V\left(\mathcal{B}_{0}\right)$, such that the families $\mathcal{B}_{i}=\mathcal{B}_{0}-\cup_{j=0}^{i-1} S_{j}$ for $i=1, \ldots, t$ satisfy

(1) $S_{i} \in \mathcal{B}_{i}$ and $\operatorname{deg}_{\mathcal{B}_{i}}\left(S_{i}\right)<d-1$ for every $i<t$,

(2) $\operatorname{deg}_{\mathcal{B}_{t}}(S) \geq d-1$ for every $S \in \mathcal{B}_{t}$.

Proof. For $i \geq 0$, if there exists a $T \in \mathcal{B}_{i}$ with $\operatorname{deg}_{\mathcal{B}_{i}}(T)<d-1$, then set $S_{i}=T$. Form $\mathcal{B}_{i+1}$ and repeat for $i+1$. If there is no such $T$, then set $i=t$ and stop.

For $n \geq 2 r-1$, we proceed by induction on $n$. The base case $n=2 r-1$ has been proved in Part I. Suppose that $n \geq 2 r,|\mathcal{A}|=\left(\begin{array}{c}n-1 \\ r-1\end{array}\right)$, and $\mathcal{A}$ contains no nontrivial intersecting family of size $d+1$. We will prove that $\mathcal{A}=X_{y}^{(r)}$ for some $y \in X$. This implies that if $\mathcal{A}^{\prime} \subset X^{(r)}$ contains no nontrivial intersecting family of size $d+1$, then $\left|\mathcal{A}^{\prime}\right| \leq\left(\begin{array}{l}n-1 \\ r-1\end{array}\right)$, by the following argument: If $\left|\mathcal{A}^{\prime}\right|>\left(\begin{array}{l}n-1 \\ r-1\end{array}\right)$, then $\mathcal{A}^{\prime}$ contains an $r$-set $R$ in addition to $X_{y}^{(r)}$ by our assumption. Now consider the subfamily consisting of all $r$-sets of $X_{y}^{(r)}$ intersecting $R$ as well as $R$ itself. This is clearly a nontrivial intersecting family, and it has size

$$
1+\left(\begin{array}{c}
n-1 \\
r-1
\end{array}\right)-\left(\begin{array}{c}
n-1-r \\
r-1
\end{array}\right)>1+r>d+1
$$

Consequently, $\left|\mathcal{A}^{\prime}\right| \leq\left(\begin{array}{c}n-1 \\ r-1\end{array}\right)$ as claimed.

Our approach is to show that there exists a vertex $x \in X$ with $\operatorname{deg}_{\mathcal{A}}(x) \leq\left(\begin{array}{c}n-2 \\ r-2\end{array}\right)$. Subsequently, the family $\mathcal{A}-\{x\}$ has size at least $\left(\begin{array}{c}n-1 \\ r-1\end{array}\right)-\left(\begin{array}{l}n-2 \\ r-2\end{array}\right)=\left(\begin{array}{l}n-2 \\ r-1\end{array}\right)$. By induction, equality holds and $\mathcal{A}-\{x\}=X_{y}^{(r)}$ for some $y \in X$; it is easy to see that every set in $\mathcal{A}$ containing $x$ also contains $y$ and $\mathcal{A}$ is the required family. Let us show that $\operatorname{deg}(x) \leq\left(\begin{array}{c}n-2 \\ r-2\end{array}\right)$ if $\left|\mathcal{L}_{x}\right|$ is a maximum.

Claim 1. $\left|\mathcal{L}_{x}\right|>\left(\begin{array}{c}n-3 \\ r-2\end{array}\right)$.

Proof. Note that $r|\mathcal{A}|=\sum_{y} \operatorname{deg}(y)=\sum_{y}\left|\mathcal{S}_{y}\right|+\sum_{y}\left|\mathcal{L}_{y}\right|$. By the choice of $x$, this is at most $|\mathcal{S}|+n\left|\mathcal{L}_{x}\right|$. Also, $\mathcal{S} \cap \mathcal{L}_{x}=\emptyset$, so $|\mathcal{S}| \leq\left(\begin{array}{c}n \\ r-1\end{array}\right)-\left|\mathcal{L}_{x}\right|$. Consequently

$$
(n-1)\left|\mathcal{L}_{x}\right| \geq r|\mathcal{A}|-\left(\begin{array}{c}
n \\
r-1
\end{array}\right)=r\left(\begin{array}{c}
n-1 \\
r-1
\end{array}\right)-\left(\begin{array}{c}
n \\
r-1
\end{array}\right)>(n-1)\left(\begin{array}{c}
n-3 \\
r-2
\end{array}\right),
$$

where the last inequality follows from a short computation and the fact that $r \geq 4$. Dividing by $n-1$, we obtain $\left|\mathcal{L}_{x}\right|>\left(\begin{array}{l}n-3 \\ r-2\end{array}\right)$.

Applying Lemma 8 to $\mathcal{L}_{x}=\mathcal{B}_{0}$, let $\left(S_{0}, S_{1}, \ldots, S_{t-1}\right)$ be the sets in $V\left(\mathcal{L}_{x}\right)$ satisfying (1) and (2), and let $\mathcal{B}_{i}$ be as in Lemma 8 . Note that $\mathcal{B}_{t} \neq \emptyset$, since otherwise

$$
\left|\mathcal{L}_{x}\right| \leq \sum_{i=0}^{t-1}\left(\operatorname{deg}_{\mathcal{B}_{i}}\left(S_{i}\right)+1\right) \leq t(d-1) \leq \frac{n-1}{r-1}(r-2)<n-1,
$$


contradicting Claim 1. Let $\mathcal{K}_{0}, \mathcal{K}_{1}, \ldots, \mathcal{K}_{s}$ be the components of $\mathcal{B}_{t}$. We let $\mathcal{K}_{i}^{\prime}$ denote the union of $\mathcal{K}_{i}$ and the family of all sets in $\mathcal{S}_{x}$ intersecting $V\left(\mathcal{K}_{i}\right)$.

Claim 2. The family $\mathcal{K}_{i}^{\prime}$ is an intersecting family.

Proof. Suppose, for a contradiction, that $\mathcal{K}_{i}^{\prime}$ contains disjoint sets $A_{0}, B_{0}$. Since $\mathcal{K}_{i}$ is connected, $\mathcal{K}_{i} \cup\left\{A_{0}, B_{0}\right\}$ is also connected. Choose a path $A_{0}, A_{1}, A_{2}, \ldots, B_{0}$ in $\mathcal{K}_{i} \cup\left\{A_{0}, B_{0}\right\}$ (possibly $A_{2}=B_{0}$ ). Then $A_{1} \in \mathcal{K}_{i}$. Lemma 8 part (2) implies that $\operatorname{deg}_{\mathcal{K}_{i}}\left(A_{1}\right) \geq d-1$, hence (if $d \geq 4$ ) there exist sets $C_{1}, C_{2}, \ldots, C_{d-3} \in \mathcal{K}_{i} \backslash\left\{A_{0}, A_{2}\right\}$ each of which intersects $A_{1}$. By definition of $\mathcal{L}_{x}$, there exists $y \in X \backslash\{x\}$ such that $A_{1} \in \mathcal{L}_{y}$. Consequently,

$$
\left\{A_{0} \cup x, A_{1} \cup x, A_{2} \cup x, C_{1} \cup x, \ldots, C_{d-3} \cup x, A_{1} \cup y\right\}
$$

is a non-trivial intersecting family of size $d+1$ in $\mathcal{A}$, since $A_{0} \cap A_{2}=\emptyset$, a contradiction.

Claim 3. $\mathcal{L}_{x}=\mathcal{K}_{0}$ and $n\left(\mathcal{L}_{x}\right) \geq n-2$.

Proof. We first show $t=s=0$, so that $\mathcal{L}_{x}=\mathcal{K}_{0}$. For a contradiction, suppose $t>0$ or $s>0$. By Claim $2, \mathcal{K}_{i} \subset \mathcal{K}_{i}^{\prime}$ is an intersecting family of $(r-1)$-sets. Therefore, for $n\left(\mathcal{K}_{i}\right) \geq 2(r-1)$, the Erdős-Ko-Rado theorem shows $\left|\mathcal{K}_{i}\right| \leq\left(\begin{array}{c}n\left(\mathcal{K}_{i}\right)-1 \\ r-2\end{array}\right) \leq\left(\begin{array}{c}n\left(\mathcal{K}_{i}\right) \\ r-2\end{array}\right)$. If $n\left(\mathcal{K}_{i}\right) \leq 2 r-3$, then $\left|\mathcal{K}_{i}\right| \leq\left(\begin{array}{c}n\left(\mathcal{K}_{i}\right) \\ r-1\end{array}\right)$, and this is at most $\left(\begin{array}{c}n\left(\mathcal{K}_{i}\right) \\ r-2\end{array}\right)$. Since $n\left(\mathcal{K}_{i}\right) \geq r-1$ for $i \leq s$, convexity of binomial coefficients yields

$$
\sum_{i=0}^{s}\left|\mathcal{K}_{i}\right| \leq \sum_{i=0}^{s}\left(\begin{array}{c}
n\left(\mathcal{K}_{i}\right) \\
r-2
\end{array}\right)=\left(\begin{array}{c}
n\left(\mathcal{K}_{0}\right) \\
r-2
\end{array}\right)+\sum_{i=1}^{s}\left(\begin{array}{c}
n\left(\mathcal{K}_{i}\right) \\
r-2
\end{array}\right) \leq\left(\begin{array}{c}
{\left[\sum_{i=0}^{s} n\left(\mathcal{K}_{i}\right)\right]-s(r-1)} \\
r-2
\end{array}\right)+\sum_{i=1}^{s}\left(\begin{array}{l}
r-1 \\
r-2
\end{array}\right) .
$$

Recalling that $n\left(\mathcal{L}_{x}\right)=\sum_{i=0}^{s} n\left(\mathcal{K}_{i}\right)+t(r-1)$, we obtain

$$
\sum_{i=0}^{s}\left|\mathcal{K}_{i}\right| \leq\left(\begin{array}{c}
n\left(\mathcal{L}_{x}\right)-s(r-1)-t(r-1) \\
r-2
\end{array}\right)+s(r-1) .
$$

By the argument giving the first two inequalities of (2), and $d-1 \leq r-1$, we have

$$
\left|\mathcal{L}_{x}\right|=\sum_{i=0}^{s}\left|\mathcal{K}_{i}\right|+\sum_{i=0}^{t-1}\left(\operatorname{deg}_{\mathcal{B}_{i}}\left(S_{i}\right)+1\right) \leq\left(\begin{array}{c}
n\left(\mathcal{L}_{x}\right)-(s+t)(r-1) \\
r-2
\end{array}\right)+s(r-1)+t(r-1) .
$$

If $s+t \geq 1$ then, by convexity of binomial coefficients,

$$
\left|\mathcal{L}_{x}\right| \leq\left(\begin{array}{c}
n\left(\mathcal{L}_{x}\right)-(r-1) \\
r-2
\end{array}\right)+(r-1) \leq\left(\begin{array}{c}
n-r \\
r-2
\end{array}\right)+(r-1)
$$


As $n \geq 2 r$ and $r \geq 4$, this contradicts Claim 1. Thus $s=t=0$, and $\mathcal{L}_{x}$ consists of one component, $\mathcal{K}_{0}$.

We now show that $n\left(\mathcal{L}_{x}\right) \geq n-2$. By the arguments above, $\left|\mathcal{K}_{0}\right| \leq\left(\begin{array}{c}n\left(\mathcal{K}_{0}\right) \\ r-2\end{array}\right)$. Therefore, by Claim $1, n\left(\mathcal{K}_{0}\right)=n\left(\mathcal{L}_{x}\right) \geq n-2$. This completes the proof of Claim 3 .

We now complete Part III and the proof of Theorem 2, by showing that $\operatorname{deg}(x) \leq\left(\begin{array}{l}n-2 \\ r-2\end{array}\right)$. By Claim $2, \mathcal{K}_{0}^{\prime}$ is an intersecting family. Since $n\left(\mathcal{K}_{0}\right) \geq n-2>n-r+1, \operatorname{tr}(x)=\mathcal{K}_{0}^{\prime}$ so $\operatorname{tr}(x)$ is itself an intersecting family of $(r-1)$-sets. As $n-1 \geq n\left(\mathcal{K}_{0}^{\prime}\right) \geq n\left(\mathcal{K}_{0}\right) \geq n-2 \geq 2(r-1)$, the Erdős-Ko-Rado theorem implies that

$$
\operatorname{deg}(x)=\left|\mathcal{K}_{0}^{\prime}\right|=|\operatorname{tr}(x)| \leq\left(\begin{array}{l}
n-2 \\
r-2
\end{array}\right) .
$$

This completes the proof of Theorem 2 .

\section{Proof of Theorem 3.}

Part III of the proof of Theorem 2 can be extended to the case $r=3$ and $2 \leq d \leq 6$ by addition of some technical details. However, Chvátal [3] and Frankl and Füredi [11] already settled the case $r=3$ and $d=2$ so we do not consider this case here. In fact, from the proof below, it follows that for $2 \leq d \leq 6$ and $n \geq 15$, a family $\mathcal{A} \subset X^{(3)}$ containing no non-trivial intersecting family of size $d+1$ has at most $\left(\begin{array}{c}n-1 \\ 2\end{array}\right)$ members, with the equality as in Theorem 2 .

We now prove Theorem 3, employing the weight counting methods of Frankl and Füredi.

Proof of Theorem 3. Let $\mathcal{A} \subset X^{(3)}$ and suppose $\mathcal{A}$ contains no non-trivial intersecting family of size $d+1$. Following Frankl and Füredi, the weight of a set $A \in \mathcal{A}$ is defined by

$$
\omega(A)=\sum_{\{x, y\} \subset A} \frac{1}{|\operatorname{tr}\{x, y\}|} .
$$

Then

$$
\begin{aligned}
\sum_{A \in \mathcal{A}} \omega(A) & =\sum_{A \in \mathcal{A}} \sum_{\{x, y\} \subset A} \frac{1}{|\operatorname{tr}(x, y)|} \\
& \leq \sum_{\{x, y\} \in X} \sum_{\substack{A \in \mathcal{A} \\
\{x, y\} \in A}} \frac{1}{|\operatorname{tr}(x, y)|} \leq \sum_{\{x, y\} \in X} 1=\left(\begin{array}{c}
n \\
2
\end{array}\right) .
\end{aligned}
$$

Equality holds if and only if every pair in $X$ is contained in some set in $\mathcal{A}$. 
As $\mathcal{A}$ contains no non-trivial intersecting family of size $d+1,|\operatorname{tr}\{x, y\}|=1$ for some $\{x, y\} \in A$ or $\sum_{x, y \in A}|\operatorname{tr}\{x, y\}| \leq d+2$. This implies that for all $A \in \mathcal{A}$,

$$
\omega(A) \geq \min \left\{1+\frac{2}{n-2},\left\lfloor\frac{(d+2)}{3}\right\rfloor^{-1}+\left\lfloor\frac{(d+3)}{3}\right\rfloor^{-1}+\left\lfloor\frac{(d+4)}{3}\right\rfloor^{-1}\right\} .
$$

For $d \geq 7$, the second term is smaller (in fact, less than 1 ). Therefore

$$
\sum_{A \in \mathcal{A}} \omega(A) \geq\left(\left\lfloor\frac{(d+2)}{3}\right\rfloor^{-1}+\left\lfloor\left[\frac{(d+3)}{3}\right\rfloor^{-1}+\left\lfloor\frac{(d+4)}{3}\right\rfloor^{-1}\right)|\mathcal{A}|\right.
$$

Together with $\sum_{A \in \mathcal{A}} \omega(A) \leq\left(\begin{array}{l}n \\ 2\end{array}\right)$, this gives the upper bound on $|\mathcal{A}|$ in Theorem 3, which is for $d \geq 7$.

For the lower bound in Theorem 3, it suffices to show that every non-trivial intersecting family of size $d+1 \geq 11$ contains a pair in at least $\left\lceil\frac{d}{3}\right\rceil$ of its edges. Then a Steiner $\left(n, 3,\left\lceil\frac{d}{3}\right\rceil-1\right)$-system, for those $n$ for which such a structure exists, does not contain such an intersecting family.

Lemma 9 Let $\mathcal{F} \subset X^{(3)}$ be a non-trivial intersecting family with $|\mathcal{F}| \geq 11$. Then there exist distinct elements $x, y \in X$ such that

$$
\left|\operatorname{tr}_{\mathcal{F}}\{x, y\}\right| \geq \frac{1}{3}(|\mathcal{F}|-1)
$$

Proof. For $a, b \in X$, we let $d(a, b)=\left|\operatorname{tr}_{\mathcal{F}}\{a, b\}\right|$. First suppose that there exist $x, y \in X$ with $d(x, y) \geq 3$. Now let $u, v, w \in \operatorname{tr}_{\mathcal{F}}\{x, y\}$. Throughout the proof, we assume $d(x, y) \leq$ $\left\lceil\frac{1}{3}(|\mathcal{F}|-1)\right\rceil-1$, otherwise we are done. Let $L_{x}=\operatorname{tr}_{\mathcal{F}}(x)-\{y\}=\left\{A \in \operatorname{tr}_{\mathcal{F}}(x): y \notin A\right\}$ and let $L_{y}=\operatorname{tr}_{\mathcal{F}}(y)-\{x\}=\left\{A \in \operatorname{tr}_{\mathcal{F}}(y): x \notin A\right\}$ Since $\mathcal{F}$ is an intersecting family,

$$
A \cap B \neq \emptyset \text { for every } A \in L_{x} \text { and } B \in L_{y} \quad(*) .
$$

Case 1: $L_{x}$ contains a matching of size three.

In this case, $L_{x}$ consists of three stars with distinct centers in $X$. By $(*)$, every pair in $L_{y}$ intersects all three centers. This implies $L_{y}=\emptyset$. As $\mathcal{F}$ is a non-trivial intersecting family, there is a triple in $\mathcal{F}$ disjoint from $x$. Since $L_{y}=\emptyset$ and $d(x, y) \geq 3$, this triple must be $\{u, v, w\}$ and $d(x, y)=3$. Since $\mathcal{F}$ is intersecting, the centers of the three stars must also be $u, v, w$. Now every $F \in \mathcal{F}-\{y\}$ with $F \neq\{u, v, w\}$ contains $x$. Therefore, assuming $d(x, u) \geq d(x, v) \geq d(x, w)$, we find 


$$
d(x, u) \geq \frac{1}{3}(|\mathcal{F}|-4)+1=\frac{1}{3}(|\mathcal{F}|-1) .
$$

This completes the proof in Case 1.

Case 2: $L_{x}$ and $L_{y}$ contain no matching of size three.

It is not hard to see by $(*)$ that $\left|L_{x}\right|+\left|L_{y}\right| \leq 2\left(\left\lceil\frac{1}{3}(|\mathcal{F}|-1)\right\rceil-1\right)+1$, with equality if and only if $L_{x}$ consists of a pair of stars of size $\lceil(|\mathcal{F}|-1) / 3\rceil-1$ with distinct centers $a, b$ and $L_{y}$ consists of the pair $\{a, b\}$. Then $|\mathcal{F}|-\left|L_{x}\right|-\left|L_{y}\right|-d(x, y) \geq 2$, unless $|\mathcal{F}|=3 k+2, k \geq 3$ and $L_{x}$ and $L_{y}$ are as described above. By $(*)$, any triple in $\mathcal{F}-\{x, y\}$ contains $u, v$ and $w$. This shows $|\mathcal{F}-\{x, y\}|=1$, and therefore $|\mathcal{F}|=3 k+2, k \geq 3$. In this case, $\{u, v, w\} \in \mathcal{F}$ and $a, b \in\{u, v, w\}$, since $\mathcal{F}$ is intersecting. Therefore $d(a, x) \geq 4$ (and also $d(b, x) \geq 4$ ), completing the proof in Case 2 .

If every pair $a, b \in X$ has $d(a, b) \leq 2$, then the arguments in Cases 1 and 2 still apply to give a contradiction with $|\mathcal{F}| \geq 11$, since in this case $|\mathcal{F}|=8$. This completes the proof of the Lemma.

\section{Proof of Theorem 7.}

Let $\mathcal{A}$ be a family of subsets of $X$ containing no non-trivial intersecting family of size $d+1$. We prove Theorem 7 by showing that $\mathcal{A}^{\prime}=\mathcal{A} \cap X^{(\geq 2)}$ has size at most $2^{n-1}-1$, with equality if and only if $\mathcal{A}^{\prime}=X_{x}^{(\geq 2)}$ for some $x \in X$. Theorem 7 is proved in two parts. Part I deals with the case $d=2$, by induction on $n \geq 1$. In Part II, we use Part I to prove Theorem 7 for $d \geq 3$.

\section{Part I: $d=2$}

Theorem 7 is easily verified for $n \leq 3$. Now let $n \geq 4$ and $w \in X$.

Case 1: For every partition of $X \backslash\{w\}$ into two non-empty sets $Y$ and $Z$, there exists a set $A \in \mathcal{A}^{\prime}-\{w\}$ such that $A \cap Y \neq \emptyset$ and $A \cap Z \neq \emptyset$. Then, for each partition of $X \backslash\{w\}$ into sets $Y$ and $Z$, either $Y \cup\{w\} \notin \mathcal{A}$ or $Z \cup\{w\} \notin \mathcal{A}^{\prime}$ - otherwise $\mathcal{A}^{\prime}$ contains a triangle. Therefore $\operatorname{deg}_{\mathcal{A}^{\prime}}(w) \leq 2^{n-2}$. By induction, $\mathcal{A}^{\prime \prime}=\mathcal{A}^{\prime}-\{w\}$ has size at most $2^{n-2}-1$, with equality if and only if $\mathcal{A}^{\prime \prime}=(X \backslash\{w\})_{x}^{(\geq 2)}$ for some $x \in X-\{w\}$. Thus

$$
\left|\mathcal{A}^{\prime}\right|=\operatorname{deg}_{\mathcal{A}^{\prime}}(w)+\left|\mathcal{A}^{\prime \prime}\right| \leq 2^{n-2}+\left(2^{n-2}-1\right)=2^{n-1}-1 .
$$


Now suppose that equality holds above. We will show that every set in $\mathcal{A}^{\prime}$ containing $w$ also contains $x$. Suppose on the contrary that $w \in S \in \mathcal{A}^{\prime}$ and $x \notin S$. Among all such $S$, choose the one of minimum size, call it $S_{0}$. Let $T$ be another set containing $w$. By the choice of $S_{0}$, either $T \supset S_{0}$, or there exist $t \in T-S$, and $s \in S-T$ (possibly $t=x$ ). In the latter case, $\{x, s, t\}, S, T$ form a triangle (replace $\{x, s, t\}$ by $\{s, t\}$ if $t=x$ ). We may therefore assume that every set in $\mathcal{A}^{\prime}$ containing $w$ also contains $S_{0}$. Hence $2^{n-2}=\operatorname{deg}_{\mathcal{A}^{\prime}}(w) \leq 2^{n-\left|S_{0}\right|-1}$ from which we conclude that $S_{0}=\{s\}$, and $E \cup\{w\} \in \mathcal{A}^{\prime}$ for every $E \subset X \backslash\{w, s\}$. Since $|X| \geq 4$, there exist distinct $a, b$ for which $\{w, s, a\}$ and $\{w, s, b\}$ lie in $\mathcal{A}^{\prime}$. Together with $\{x, a, b\}$ (or just $\{a, b\}$ if $a=x$ or $b=x$ ) this once again forms a triangle.

Case 2: There exists a partition of $X \backslash\{w\}$ into two nonempty sets $Y$ and $Z$ such that no member of $\mathcal{A}^{\prime}$ in $X \backslash\{w\}$ contains an element of both $Y$ and $Z$. By induction, at most $2^{|Y|}-1$ elements of $\mathcal{A}^{\prime}$ are contained in $Y \cup\{w\}$, and similarly for $Z$. The number of sets which contain an element of $Y$ and an element of $Z$ is, by the choice of $Y$ and $Z$, at most $2^{n-1}-1-\left(2^{|Y|}-1\right)-\left(2^{|Z|}-1\right)$. Therefore

$$
\left|\mathcal{A}^{\prime}\right| \leq\left(2^{|Y|}-1\right)+\left(2^{|Z|}-1\right)+\left(2^{n-1}-1-\left(2^{|Y|}-1\right)-\left(2^{|Z|}-1\right)\right)=2^{n-1}-1 .
$$

If equality holds, then by induction there exist $y \in Y \cup\{w\}, z \in Z \cup\{w\}$ with $(Y \cup\{w\})_{y}^{(\geq 2)} \cup$ $(Z \cup\{w\})_{z}^{(\geq 2)} \subset \mathcal{A}^{\prime}$. Since $|X| \geq 4$, we may assume by symmetry that $|Y| \geq 2$. We next show that $y=w$. Observe that for every set $S \subset X \backslash\{w\}$ containing an element of both $Y$ and $Z$, we have $S \cup\{w\} \in \mathcal{A}^{\prime}$. If $y \neq w$, then $\{y, a\} \in \mathcal{A}^{\prime}$ for some $a \in Y \backslash\{y\}$. The set $\{y, a\}$ together with $\{y, w\}$ and $\{w, a, b\}$ for some $b \in Z$ forms a triangle. Consequently $y=w$, and $z=w$ as well unless $Z=\{z\}$. But in this case $(Z \cup\{w\})_{z}^{(\geq 2)}=(Z \cup\{w\})_{w}^{(\geq 2)}$, therefore $\mathcal{A}^{\prime}=X_{w}^{(\geq 2)}$.

\section{Part II: $d \geq 3$}

Define a function $f$ on the positive integers by $f(1)=f(2)=f(3)=1$, and for $n \geq 4$,

$$
f(n)=\max \left\{0, f(n-3)+d-2^{n-4}\right\} .
$$

It is easy to see that if $n \geq 4$, and $f(n) \geq 0$, then

$$
f(n)=1+\left(\left\lceil\frac{n}{3}\right\rceil-1\right) d-\sum_{i=0}^{\lfloor(n-4) / 3\rfloor} 2^{n-4-3 i} .
$$

Set $n_{d}=\log _{2} d+\log _{2} \log _{2} d+2$. An easy calculation now shows that $f(n)=0$ whenever $n \geq n_{d}$ and $f(n)>f(n-3)+d-2^{n-4}$ when $n>n_{d}$. 
In this part of the proof, we proceed by induction on $n \geq 1$, with the following hypothesis: Let $\mathcal{A}^{\prime} \subset X^{(\geq 2)}$ contain no non-trivial intersecting family of size $d+1$. Then $\left|\mathcal{A}^{\prime}\right| \leq 2^{n-1}-1+f(n)$.

For $n \leq 3$, the result is true as

$$
\left|\mathcal{A}^{\prime}\right| \leq\left|X^{(\geq 2)}\right|=2^{n}-n-1 \leq 2^{n-1}-1+f(n) .
$$

Now suppose that $n \geq 4$. By Part I, we may assume $\mathcal{A}^{\prime}$ contains a triangle $\mathcal{F}=\left\{F_{1}, F_{2}, F_{3}\right\}$, otherwise the proof is complete.

Let $x, y, z$ be elements in $F_{1} \cap F_{2}, F_{2} \cap F_{3}$ and $F_{3} \cap F_{1}$ respectively. Then at most $d$ sets in $\mathcal{A}^{\prime}$ intersect $\{x, y, z\}$ in at least two points, otherwise $\mathcal{F}$ together with another $d-2$ of these sets forms a non-trivial intersecting family of size $d+1$. The total number of sets in $\mathcal{A}^{\prime}$ intersecting $\{x, y, z\}$ is therefore at most $3 \cdot 2^{n-3}+d$. Let $\mathcal{A}^{\prime \prime}=\mathcal{A}^{\prime}-\{x, y, z\}$. Then

$$
\left|\mathcal{A}^{\prime}\right| \leq\left|\mathcal{A}^{\prime \prime}\right|+3 \cdot 2^{n-3}+d
$$

As $\mathcal{A}^{\prime \prime}$ contains no non-trivial family of size $d+1$, the induction hypothesis shows $\left|\mathcal{A}^{\prime \prime}\right| \leq 2^{n-4}$ $1+f(n-3)$. This gives

$$
\begin{aligned}
\left|\mathcal{A}^{\prime}\right| & \leq 2^{n-4}-1+f(n-3)+3 \cdot 2^{n-3}+d \\
& =2^{n-1}-1+f(n)-\left(f(n)-f(n-3)-d+2^{n-4}\right) \\
& \leq 2^{n-1}-1+f(n), \quad(* *)
\end{aligned}
$$

where the last inequality follows from $(*)$. By the choice of $n_{d}$, we know that $f(n)=0$ for $n \geq n_{d}$, so $\left|\mathcal{A}^{\prime}\right| \leq 2^{n-1}-1$ for $n \geq n_{d}$, completing the proof of the upper bound in Theorem 7 .

Now suppose that $\left|\mathcal{A}^{\prime}\right|=2^{n-1}-1$ and $n>n_{d}$. Then the inequality $(* *)$ is strict. This gives the contradiction $\left|\mathcal{A}^{\prime}\right|<2^{n-1}-1$. Consequently $\mathcal{A}^{\prime}$ contains no triangle and Part I of the proof applies to give the case of equality.

\section{Acknowledgments}

Most of the work for this paper was done while both authors were Postdoctoral Researchers in the Theory Group at Microsoft Research. The authors also thank a referee for very helpful comments and minor corrections on an earlier draft, and Yi Zhao for carefully reading the proof of Theorem 7. 


\section{References}

[1] Baranyai, Zs., On the factorization of the complete uniform hypergraph. Infinite and finite sets (Colloq., Keszthely, 1973; dedicated to P. Erdős on his 60th birthday), Vol. I, pp. 91-108. Colloq. Math. Soc. Janos Bolyai, 10 North-Holland, Amsterdam, 1975.

[2] Bermond, J.-C., Frankl, P., On a conjecture of Chvátal on $m$-intersecting hypergraphs. Bull. London Math. Soc. 9 (1977), no. 3, 310-312.

[3] Chvátal, V., An extremal set-intersection theorem. J. London Math. Soc. (2) 9 (1974/75), $355-359$.

[4] Bollobás, B., Duchet, P., On Helly families of maximal size. J. Combin. Theory Ser. B 35 (1983), no. 3, 290-296.

[5] Bollobás, B., Duchet, P., Helly families of maximal size. J. Combin. Theory Ser. A 26 (1979), no. 2, 197-200.

[6] Csákány, R., Kahn, J., A homological approach to two problems on finite sets. (English. English summary) J. Algebraic Combin. 9 (1999), no. 2, 141-149.

[7] Erdős, P., Topics in combinatorial analysis. Proc. Second Louisiana Conf. on Comb., Graph Theory and Computing (R. C. Mullin et al., Eds.) pp 2-20, Louisiana State Univ., Baton Rouge 1971.

[8] Erdős, P., Ko, H., Rado R., Intersection theorems for systems of finite sets. Quart. J. Math. Oxford Ser. (2) 12 (1961) 313-320.

[9] Frankl, P., On Sperner families satisfying an additional condition. J. Combinatorial Theory Ser. A 20 (1976), no. 1, 1-11.

[10] Frankl, P., On a problem of Chvátal and Erdős on hypergraphs containing no generalized simplex. J. Combin. Theory Ser. A 30 (1981), no. 2, 169-182.

[11] Frankl, P., Füredi, Z., Exact solution of some Turán-type problems. J. Combin. Theory Ser. A 45 (1987), no. 2, 226-262.

[12] O. P. Lossers, P. Erdos and E. Milner: P. 190, Canad. Math Bull 16 (1973), 145-146.

[13] Mulder, H. M., The number of edges in a $k$-Helly hypergraph. Combinatorial mathematics (Marseille-Luminy, 1981), 497-501, North-Holland Math. Stud., 75, North-Holland, Amsterdam, 1983. 
[14] Ray-Chaudhuri, D., Wilson, R., The existence of resolvable block designs. Survey of combinatorial theory (Proc. Internat. Sympos., Colorado State Univ., Fort Collins, Colo., 1971), pp. 361-375. North-Holland, Amsterdam, 1973.

[15] Tuza, Zs., Helly-type hypergraphs and Sperner families. European J. Combin. 5 (1984), no. $2,185-187$.

[16] Tuza, Zs., Helly property in finite set systems. J. Combin. Theory Ser. A 62 (1993), no. 1, $1-14$. 